anterior wound, after which the other viscera were inspected. No further injury being manifest, the abdomen was subjected to a thorough cleansing with sterile gauze wrung out of sterile salt solution and a free washing with hot sterile salt solution. Lamp-wick drainage was introduced under the bile ducts and under the posterior surface of the stomach and the wound was closed by through and through sutures and the usual sterile dressing applied.

The operation lasted about one hour and fifteen minutes, being prolonged by such operative diffisulties as tympany of the intestines and poor light.

In the search for injury to other viscera we were unable to trace the bullet after it left the stomach. After the operation was concluded, the patient was put to bed in fairly good condition.

Subsequent History.-Nothing eventful occurred until the second day, when the pulse became rapid, though the temperature was practically normal; a marked jaundice was manifest. At the end of the first twenty-four hours after the operation the first dressing was made. At this time the entire dressing was saturated with secretions carried out by our lamp-wick drainage. At the end of the second twentyfour hours, the sesond dressing was made and the lamp-wick drainage removed. This dressing was also fairly well saturated with secretions. On the third day the jaundice was more marked. On the fourth day, the jaundice began to fade and had about all disappeared at the end of the first week. During the first week, the patient was fed by nutritive enemata and the saline solution was continued through the first ten days, nothing being administered by the mouth, exoept occasional teaspoonsful of iced water. During the first ten days, the temperature ranged from 97 to $101 \mathrm{~F}$. This temperature was largely accounted for by the infection of the abdominal stitches. These stitches were placed under more than ordinary tension, as the patient was very much bloated at the time of the operation and the abdominal eavity was further distended to its full capacity by saline solution.

After the tenth day the patient was fed with milk and lime water and predigested foods, plenty of liquids being administered at regular intervals. This form of nutrition was continued up to the fourteenth day, after which time he was allowed a liberal diet of light foods, both solid and liquid, and all the fluids that he desired.

During the second week the patient developed a marked dyspnea which was manifest by shortness of breath, a moderate cough and a pain in the right side on breathing and coughing. There was no spitting of blood or rusty sputum, but examination revealed consolidation of the lower lobe of the right lung and of the posterior surface of the middle lobe extending as high as the superior border of the scapula.

These conditions remained practically unchanged up to the twenty-first day, except that there was an increased temperature and pulse rate until the night of the twenty-first day, when he had a profuse sweat and the temperature fell to normal. Pain left and breathing became quite natural, this evidently being the crisis, which came suddenly and was very marked.

After this the lung conditions remained very obstinate as far as clearing and restitution of function were concerned, it being a very marked and stubborn case of delayed resolution. By the fifth week, however, the lungs were fairly clear and the patient steadily improving. This progress continued up to the forty-eighth day, at which time he was able to take short walks and to take care of himself very nicely, and was discharged from the hospital on this day.

Remarks. - I believe that drainage was of inestimable service in this case. The patient had comparatively thin abdominal wall and a moderately fat omentum, and this, together with the median incision, very much lessened the operative difficulties of seeing and treating the doyble rent in the stom. ach and gave ample room for inspecting other viscera and cleaning the wound.

The treatment of gun-slot wounds of the stomach as practiced by military surgeons in the field, which is shown to be uniformly unsuccessful, has led many physicians to believe that laparotomy is not indicated in these injuries, and prob- ably some lives have been sacrificed by not treating them promptly by operation. This, however, should not be undertaken unless surroundings are favorable for clean and aseptic work.

I am indebted to Drs. Frank Miller and C. E. MeCauley for valuable assistance and suggestions in the treatment and aftercare of the patient. I am also indebted to the Sisters of St. Luke's Hospital for special care and preparation of the patient.

\section{CHRONIC PURPURA HEMORRHAGICA.}

\section{H. H. LOVELAAND, M.D. SYRACUSE, N. $\mathrm{Y}$.}

The editorial statement in Tire Journal, June 10, that chronic forms of purpura hemorrhagica are so seldom mentioned in medical literature that we are apt to regard them as being exceedingly rare, leads me to report a case that came under my observation during the years 1896 to 1903, inclusive.

Patient.-C. D., male, was born May or June, 1895, date not certain. His parents were of German birth, and both of them exceptionally large people, each weighing over 200 pounds, but well proportioned and in vigorous health. This child was one of twelve, five of whom died in infancy, or in very early years, the others being in excellent health.

History.-I first saw him with the physician who had at tended him from birth, in December, 1895, and the beginning of the following year he came under my care and remained so till October, 1903. He was a large, well-formed, fat baby of 6 or 7 months. He was somewhat anemic, but there was no discoloration, sponginess or hemorrhage of the gums, no swelling of the thighs, or any of the classical signs of scorbutus. The mother was nursing him. She had plenty of milk, of a good quality. He was nursed until 18 months of age, the last six or seven months potatoes and other foods also being used. As the child developed he ate bread and butter, meat, potatoes and other vegetables, fruit, etc., the same as the rest of the family, and as they kept a cow he had plenty of good cow's milk. 1 do not know when the purpura began, but it must have been in the very early months, for I was told that he had received nearly every known treatment, but to no purpose thus far. From that time I had frequent opportunity to witness the appearance, course and disappearance of the purpuric areas. From the first date given until some four years later the child was scarcely ever without the spots somewhere on his body, varying in size from that of a dime to that of a small orange. One of the latter I saw on his forehead once, and, if I had not known the cause 1 should have thought that he had received a severe blow with consequent ecchymosis, the disappearance of the spot being the same as that from a blow-gradually turning from red to dark blue, and then greenish yellow, etc., until it faded away entirely. After his fifth year the spots appeared at gradually lengthening intervals, and the rheumatic phase of the case became more prominent. The child had severe inflammatory reaction about the joints, especially the knee and shoulder. Salicylates were administered faithfully, but apparently with no effect. He had previously had Fowler's solution, syrup of hypophosphites comp., and other tonies.

The knee became partly flexed, and in spite of all that I could do to get him to use the leg and knee or his parents to manipulate them during the intervals, he continued to go about with a partly flexed knee and used a crutch or stick to assist him in walking. His blood was thin, lacking in color, not easily coagulated, and he bled excessively from the slightest wound, though I was generally able to check hemorrhage by the use of Monsel's solution and tightly applied bandage.

The case may be considered by some as one of scurvy, but from the fact that there was no emaciation, no spongy or bleeding gums, no swelling or deformity of the thigh, leg or other bones (at least for the first five years), and also because there was no apparent reason for such trouble in the food, either as regards quality or quantity, I have not been able so to classify it. My removal from the locality has taken from my observation what was to me a very interesting case. 